\title{
Review
}

\section{Does sentinel lymph node detection play a role in patients with vaginal cancer?}

\author{
Nicolae Bacalbasa ${ }^{1,2,3}$, Irina Balescu ${ }^{4 *}$ Mihai Dimitriu ${ }^{5}$, Cristian Balalau, \\ Mihaela Vilcu ${ }^{1,2}$, Iulian Brezean ${ }^{1,2}$ \\ ${ }^{1}$ Ion Cantacuzino Clinical Hospital, Bucharest, Romania \\ ${ }^{2}$ Carol Davila University of Medicine and Pharmacy, Bucharest, Romania \\ ${ }^{3}$ Center of Excellence in Translational Institute, Fundeni Clinical Institute, Bucharest, Romania \\ ${ }^{4}$ Ponderas Academic Hospital, Bucharest, Romania \\ ${ }^{5}$ St. Pantelimon Emergency Clinical Hospital, Bucharest, Romania
}

\begin{abstract}
The role of sentinel lymph node detection was clearly demonstrated in patients diagnosed with gynecologic malignancies such as breast, endometrial, cervical or vulvar cancer. Therefore, the method provides an adequate staging of the disease with minimal postoperative complications when compared to classical extended lymphadenectomy. Recently, the method has been successfully reported in vaginal cancer patients. This is a literature review of the current papers published so far on the issue of sentinel lymph node detection in vaginal cancer patients.

Although only few cases have been reported so far, it seems that sentinel lymph node biopsy in vaginal cancer patients represents a safe, effective and feasible method to diminish the rates of unnecessary extended lymph node dissection in vaginal cancer patients. However, due to the rarity of the disease in association with the low number of cases reported so far, the method is not part of the therapeutic standard protocol for this disease, larger studies still being needed.
\end{abstract}

Keywords

: sentinel lymph node biopsy, vaginal cancer, lymphadenectomy

Highlights

$\checkmark \quad$ Biopsy of sentinel lymph nodes appears to be a safe, effective and feasible method for vaginal cancer patients, able to assess the cases of unnecessary extended lymph node dissection.

$\checkmark \quad$ This method is not yet part of the standard therapeutic protocol for this disease, because it is a relatively rare disease associated with a low number of cases investigated so far.

To cite this article: Bacalbasa N, Balescu I, Dimitriu M, Balalau C, Vilcu M, Brezean I. Does sentinel lymph node detection play a role in patients with vaginal cancer? J Clin Invest Surg. 2019; 4(1): 1-4. DOI: $10.25083 / 2559.5555 / 4.1 / 1.4$ 


\section{Introduction}

Vaginal cancer represents the rarest gynecologic malignancy, with an estimated incidence lower than 0.5 cases/ 100,000 women/ year (1). Most often, these tumors prove to be squamous cell carcinomata, while a small proportion is represented by melanomata and clear cell carcinomata (2).

Very often, the first intention therapy in patients diagnosed with vaginal cancer is radiotherapy, since this approach provides a good control of the disease. In certain cases, such as patients who develop local recurrences or local complications during radiotherapy as well as in cases diagnosed with clear cell carcinomata or melanomata, surgery might represent the treatment of choice (2).

Due to the fact that cases presenting upper vaginal lesions might induce the development of pelvic lymph node metastases, while lower lesions are associated with inguinal lymph node metastases, the exact topography of the lesion is mandatory in order to orientate the irradiation field. Moreover, in cases in which surgery is the therapy of choice, performing an extended lymph node dissection at this level is associated with an increased risk of postoperative complications such as lymphoceles, lymphedema, infections or wound healing deficiencies (2).

\section{Discussions}

\section{Sentinel lymph node detection in gynecologic malignancies}

So far, performing a sentinel lymph node biopsy has become part of the standard of care in patients diagnosed with breast, cervical, endometrial and even vulvar cancer, especially when the lesion is diagnosed at an early stage. Performing sentinel lymph node biopsy provides a more efficient staging of the disease, a more adequate tailoring of the postoperative therapeutic protocol and a diminishing of the postoperative complications, such as lymphedema, lymphoceles, an inadequate wound healing or local infections $(3,4)$.

The role of sentinel lymph node detection in vaginal cancer

Due to the fact that the vagina presents similar drainage routes as the cervical and vulvar regions respectively, the method of sentinel lymph node detection was extrapolated from cervical and vulvar cancer patients. Therefore, patients diagnosed with upper half vaginal cancer are expected to metastasize especially at the level of the pelvic lymph nodes (similarly to cervical cancer patients), while cases diagnosed with lower vaginal cancer are expected to metastasize especially at the level of the inguinal lymph nodes (similarly to vulvar cancer patients). Therefore, in cases diagnosed with upper vaginal lesions traditionally, an extended pelvic lymph node dissection has been proposed, leading to the same complications as those reported in cervical cancer patients, while cases diagnosed with lower vaginal tumors are usually submitted to inguinal lymph node dissection (similarly to patients diagnosed with vulvar neoplastic disease).

However, the first studies which focused on the possible role of sentinel lymph node biopsy in vaginal cancer patients were conducted on patients diagnosed with vaginal melanoma (5-7). This aspect is obviously related to the fact that the experience in terms of sentinel lymph node biopsy in malignant melanoma is one of the largest and easily extrapolated to different locations of this malignancy, including the vaginal one.

The sentinel lymph node biopsy detection in vaginal cancer has been initially performed after the injection of radioactive markers such as Technetium -99-m nanocolloid (8). One of the first studies which demonstrated the efficacy of sentinel lymph node detection in vaginal cancer patients was conducted by Van Dam and co. and included three patients with newly diagnosed and recurrent lesions. In all cases, the radiocolloid injection was given preoperatively in four points in the peritumoral area. In the first case, the sentinel nodes were discovered in the groin and obturator regions and turned negative. In the second case, the sentinel node was found at the level of the junction of the iliac vessels and turned positive, therefore the patient was submitted to adjuvant chemoirradiation, while in the third case the sentinel lymph node was found at the level of the right obturator fossa and turned negative. Interestingly, the third patient included in this study had been initially submitted to radiochemotherapy for stage III vaginal cancer, but recurred six months after ending the oncologic treatment, so she was submitted to surgery and sentinel lymph node biopsy with curative intent. Once the negativity of the sentinel nodes was proven, the patient was submitted to a posterior exenteration with curative intent. In all cases, the sentinel lymph node was detected laparoscopically, thus minimizing the postoperative complication rates (8).

The largest experience regarding sentinel lymph node detection by using a radiocolloid injection comes from the M.D. Anderson Cancer Center, Houston, Texas, the United States of America (2). The study was conducted on 14 patients diagnosed with vaginal cancer. In 11 of the 14 cases, the sentinel lymph node was successfully identified. Among the 14 cases, the authors reported seven patients who had been previously submitted to prior hysterectomy, 
while two other cases had been previously submitted to a local resection of the tumor. The median number of identified lymph nodes was two, while in six cases bilateral sentinel lymph nodes were revealed. Interestingly, in three of the six cases with bilateral sentinel lymph nodes, the initial lesion was situated at the midline level. Another interesting point of the study was the one related to cases prior to neoadjuvant radiation therapy. Therefore, it seems that in all these cases, the lymphatic mapping as well as the lymphoscintigraphy was influenced by the distortion of the lymphatic vessels during radiotherapy (2).

One of the largest studies which tested the feasibility of sentinel lymph node biopsy in vaginal cancer patients by using a dual method of detection was conducted by Hertel and co. and published in 2013 (9). The study included seven patients submitted to vaginal cancer surgery, in all cases Technetium nanocolloid and patent blue being injected in the peritumoral area. According to the authors, the detection rate was $100 \%$, the median number of detected lymph nodes being 2.6 by using the radiocolloid injection and 4.3 by using the blue dye injection. From the seven cases introduced in the current study, the pelvic location of the sentinel nodes was encountered in six cases, all of them presenting upper or middle third vaginal lesions, while among cases presenting middle and lower third lesions the inguinal location of the sentinel nodes was encountered in four cases. Moreover, all cases presenting nodal metastases reported at least one positive sentinel lymph node, no false negative results being reported. These data enabled the authors to consider that the method is safe in order to identify which cases diagnosed with vaginal cancer should be routinely submitted to an extended lymph node dissection (9).

Recently, a new method with promising results, based on the indocyanine green injection, has been proposed. The principle of the method is based on the characteristic of this fluorescent dye to be excited in the near infrared field at a wavelength of 807 nanometers and to emit a light signal of 822 nanometers later on (1).

The utility of indocyanine green in sentinel lymph node detection in a patient with vaginal cancer was reported for the first time by the scientists from Hannover in 2017 (1). The patient, a 58-year-old woman diagnosed with lower vaginal cancer measuring $2 \mathrm{~cm}$, was injected with both Technetium radiocolloid and indocyanine green. After injecting the radioactive tracer in four points in the close proximity of the tumor, the patient was submitted to a photon emission computed tomography which revealed the presence of two inguinal foci of capture, a left and a right one. Later on, indocyanine green was injected in the same points as the radiocolloid and surgery was performed 30 minutes after the dye injection. After performing a complete tumoral resection, the sentinel lymph nodes were successfully identified by using the dual method in the right groin, the nodes presenting radioactive signal, being also positive for indocyanine green staining, while at the level of the left groin there were two nodes positive for both Technetium and indocyanine green, and one node presenting only indocyanine green capture. The final histopathological studies revealed the presence of four sentinel nodes identified by technetium and six sentinel nodes identified after the indocyanine green injection, all of them being free of disease (1). The fact that the authors discovered the presence of a sentinel node positive only for indocyanine green at the level of the left groin is explained by the fact that the lymph node detection was initially performed on the right side and later on the left side, a longer time before the detection in the left side, leading to the dissemination of the green color in the non-sentinel lymph nodes.

Another similar report came from Seoul, Korea and was published in the same year. This time the patient, a 40-yearold female diagnosed with squamous cell vaginal cancer on the posterior vaginal fornix was submitted to indocyanine green injection at 3 o'clock and 9 o'clock positions of the lesion followed by radical laparoscopic hysterectomy and vaginectomy. This time, the authors reported the presence of sentinel lymph nodes bilaterally at the level of the obturator fossa. The histopathological studies demonstrated the presence of a malignant lesion with a maximal depth of $1 \mathrm{~mm}$ and with no lymph node metastases (10).

\section{Conclusions}

Although only few cases have been reported so far, it seems that sentinel lymph node biopsy in vaginal cancer patients represents a safe, effective and feasible method to diminish the rates of unnecessary extended lymph node dissection in vaginal cancer patients. However, due to the rarity of the disease in association with the low number of cases reported so far, the method is not part of the therapeutic standard protocol for this disease, larger studies still being needed.

\section{Acknowledgements}

This work was supported by the project entitled "Multidisciplinary Consortium for Supporting the Research Skills in Diagnosing, Treating and Identifying Predictive Factors of Malignant Gynecologic Disorders", project number PN-III-P1-1.2-PCCDI2017-0833. 


\section{Conflict of interest disclosure}

There are no known conflicts of interest in the publication of this article. The manuscript was read and approved by all authors.

\section{Compliance with ethical standards}

Any aspect of the work covered in this manuscript has been conducted with the ethical approval of all relevant bodies and that such approvals are acknowledged within the manuscript.

\section{References}

1. Soergel P, Hillemanns P, Klapdor R, Jentschke M, Christgen M, Hertel, H. Sentinel lymphonodectomy in early vaginal cancer using combined near infrared fluorescence from indocyanine green and technetium$99 \mathrm{~m}$ nanocolloid - a first case report. Clinical Obstetrics, Gynecology and Reproductive Medicine 2017; 3(10): 1-3. DOI: 10.15761/COGRM.1000182

2. Frumovitz M, Gayed IW, Jhingran A, Euscher ED, Coleman RL, Ramirez PT et al. Lymphatic mapping and sentinel lymph node detection in women with vaginal cancer. Gynecol Oncol. 2008; 108(3): 478-81. PMID: 18190952, DOI: 10.1016/j.ygyno.2007.12.001

3. Schnurch HG, Ackermann S, Alt CD, Barinoff J, Boing C, Dannecker C et al. Diagnosis, Therapy and Followup Care of Vulvar Cancer and its Precursors. Guideline of the DGGG and DKG (S2k-Level, AWMF Registry Number 015/059, November 2015. Geburtshilfe Frauenheilkd 2016; 76(10): 1035-49. PMID: 27765958, DOI: 10.1055/s-0042-103728

4. Hampl M, Hantschmann P, Michels W, Hillemanns P. Validation of the accuracy of the sentinel lymph node procedure in patients with vulvar cancer: results of a multicenter study in Germany. Gynecol Oncol. 2008; 111(2): 282-8. PMID: 18804850, DOI: 10.1016/j.ygyno.2008.08.007

5. Rodier JF, Janser JC, David E, Routiot T, Ott G. Radiopharmaceutical-guided surgery in primary malignant melanoma of the vagina. Gynecol Oncol. 1999; 75(2): 308-9. PMID: 10525394, DOI: 10.1006/gyno.1999.5577

6. Abramova L, Parekh J, Irvin WP, Jr., Rice LW, Taylor PT, Jr., Anderson WA et al. Sentinel node biopsy in vulvar and vaginal melanoma: presentation of six cases and a literature review. Ann Surg Oncol. 2002; 9(9): 840-6. PMID: 12417504

7. Nakagawa S, Koga K, Kugu K, Tsutsumi O, Taketani Y. The evaluation of the sentinel node successfully conducted in a case of malignant melanoma of the vagina. Gynecol Oncol. 2002; 86(3): 387-9. PMID: 12217768

8. van Dam P, Sonnemans H, van Dam PJ, Verkinderen L, Dirix LY. Sentinel node detection in patients with vaginal carcinoma. Gynecol Oncol. 2004; 92(1): 89-92. PMID: 14751143

9. Hertel H, Soergel P, Muecke J, Schneider M, Papendorf $\mathrm{F}$, Laenger $\mathrm{F}$ et al. Is there a place for sentinel technique in treatment of vaginal cancer?: feasibility, clinical experience, and results. Int J Gynecol Cancer. 2013; 23(9): 1692-8. PMID: 24177255, DOI: 10.1097/IGC.0b013e3182a65455

10. Lee IO, Lee JY, Kim S, Kim SW, Kim YT, Nam EJ. Sentinel lymph node mapping with indocyanine green in vaginal cancer. J Gynecol Oncol. 2017; 28(4): e29. PMID: 28541627, DOI: 10.3802/jgo.2017.28.e29 